\title{
Planification économique et migration en Polynésie Française
}

Anne-Marie d'Hauteserre

\section{(2) OpenEdition}

1 Journals

Édition électronique

URL : https://journals.openedition.org/remi/300

DOI : $10.4000 /$ remi.300

ISSN : $1777-5418$

Éditeur

Université de Poitiers

\section{Édition imprimée}

Date de publication : 1 mai 2004

Pagination : 119-139

ISBN : 2-911627-36-9

ISSN : 0765-0752

\section{Référence électronique}

Anne-Marie d'Hauteserre, «Planification économique et migration en Polynésie Française », Revue européenne des migrations internationales [En ligne], vol. 20 - $\mathrm{n}^{\circ} 1$ | 2004, mis en ligne le 25 septembre 2008, consulté le 15 avril 2022. URL : http://journals.openedition.org/remi/300 ; DOI : https://doi.org/ 10.4000/remi.300

Ce document a été généré automatiquement le 15 avril 2022.

(c) Université de Poitiers 


\title{
Planification économique et migration en Polynésie Française
}

\author{
Anne-Marie d'Hauteserre
}

1 Leur insularité, leurs écosystèmes fragiles et leur vulnérabilité aux désastres naturels sont autant de causes de difficultés pour les îles de taille réduite, quelle que soit leur situation politique. L'éloignement, pour certaines d'entre elles, est un obstacle supplémentaire à leur durabilité économique et même à leur survie. Une des solutions historiques adoptée par leurs résidents a été l'émigration. Un tel mouvement, dans le Pacifique, n'était possible que parce qu'un courant migratoire contraire s'était effectué antérieurement. La Polynésie française comprend des îles occupées par des migrants polynésiens qui avaient colonisé presque tout le Pacifique oriental bien avant l'apparition des Européens dans cet océan. Ces Polynésiens avaient sans doute même atteint les côtes américaines. Ils n'ont pas attendu l'expansion coloniale européenne ni celle, globale, du capitalisme pour se déplacer. Le mode en a été circulatoire et non unidirectionnel, réduisant son effet hémorragique, du moins dans certaines îles. Les liens du Territoire avec la France permettent au gouvernement territorial siégeant à Papeete de pallier l'émigration continue par des subventions de l'État français.

2 Cet article examine l'articulation entre le plan de développement économique des archipels éloignés du gouvernement du Territoire, en coopération avec l'État français, et les circuits de migration de la Polynésie française. Les archipels éloignés ont vu leur population diminuer au cours des dernières décennies (surtout depuis 1962) et l'après CEP (Centre d'Expérimentation du Pacifique) ne permet plus de pourvoir à tous les emplois qui existaient dans le « centre » ou aux Tuamotu. Les deux gouvernements, sur la base d'un contrat de plan, cherchent à ouvrir ces archipels à l'économie globale en leur fournissant des services comme l'enseignement et la santé, des moyens de transports et de communication ultra modernes ainsi qu'un soutien logistique, et souvent financier, pour démarrer des affaires qui utilisent les ressources locales (Pacte de progrès, 1993 ; État-Territoire, 1994 ; Laventure, 1997). Un des objectifs de ce lourd investissement est d'inciter au retour dans les archipels éloignés du plus grand nombre possible de ceux qui les ont quittés et d'y maintenir ceux qui y vivent actuellement, 
ainsi que leurs enfants. La Polynésie rejoint en cela les autres pays du monde qui ont choisi de contrôler les flux migratoires. Il s'agira donc d'envisager la stabilisation géographique de la population résidente des archipels extérieurs et la réintroduction de leurs émigrés, ainsi que les problèmes nés de la nécessaire réconciliation entre des usages concurrents d'occupation de l'espace dans un site de taille réduite. Les migrations relèvent de causes multiples souvent difficiles à démêler, mais leur compréhension est cependant nécessaire si l'on veut mener à bien des décisions politiques et économiques efficaces. Ces efforts indiquent (et se fondent sur) une évaluation différente de la valeur sociale et économique des îles. L'ordre social et économique des archipels éloignés n'est pas normativement établi ni ne se maintient automatiquement. Il est constamment en état de (re)construction et de transformation. Y encourager un retour est une des voies possibles de restructuration de leur économie à l'intérieur de la Polynésie.

\section{Planification économique en Polynésie française}

Dans n'importe quel espace géographique, des inégalités ont tendance à se développer parce qu'un centre avantagé attire à lui des ressources qui augmentent sa primauté. Les acteurs économiques et sociaux de ces périphéries doivent aujourd'hui faire face à de nouveaux espaces créés par des forces globales, y compris les espaces de l'Internet. La vitalité économique de la périphérie, souvent fondée sur des activités primaires, est généralement moindre. Son plus grand handicap tient à son éloignement des centres de décisions. Son développement requiert alors l'intervention du gouvernement, mais les difficultés résident dans le fait que les activités économiques y ont des effets secondaires réduits. Toute entreprise a de multiples conséquences fonctionnellement interdépendantes que les dirigeants voudraient contrôler au bénéfice de l'économie et de la société locales.

Les contrastes de densité de peuplement rendent difficile une utilisation rationnelle des ressources, laquelle se complique avec les grandes distances : la Polynésie française occupe un domaine maritime dont la superficie est équivalente à celle de l'Europe de l'Ouest (figure 1). La diversité géographique et culturelle de ces îles posent de multiples difficultés au développement équitable de toute la région. L'émigration, qui en résulte, perpétue ce déséquilibre. Tahiti et ses îles souffrent de nombreuses formes de "dépendance " mais non du syndrome de sous-développement qui a provoqué le déplacement vers des espaces internationaux d'une grande partie de la population des autres nations polynésiennes.

5 Il existe cependant une spécificité îlienne et micro-économique due en partie à une profonde différentiation fonctionnelle de son espace économique, et surtout de son marché du travail. Cette différentiation est le résultat d'interactions entre les structures héritées de sa trajectoire économique et de son expérience historique. Le CEP, par exemple, a causé le déplacement d'un grand nombre de Polynésiens entre 1962 et 1996 mais pas nécessairement de la périphérie vers le centre : Hao et Mururoa sont dans les Tuamotu. Au sein de cette main-d'œuvre, de nombreux Polynésiens ne peuvent retrouver d'emploi dans l'économie actuelle, soit parce qu'ils n'ont que des aptitudes manuelles, soit au contraire, parce qu'ils ont trop de diplômes. La globalisation économique a, elle aussi, réduit certaines options, mais elle a décuplé les perspectives dans les domaines du tourisme et du commerce électronique. 
Figure 1 : L'étendue du territoire par rapport à l'Europe

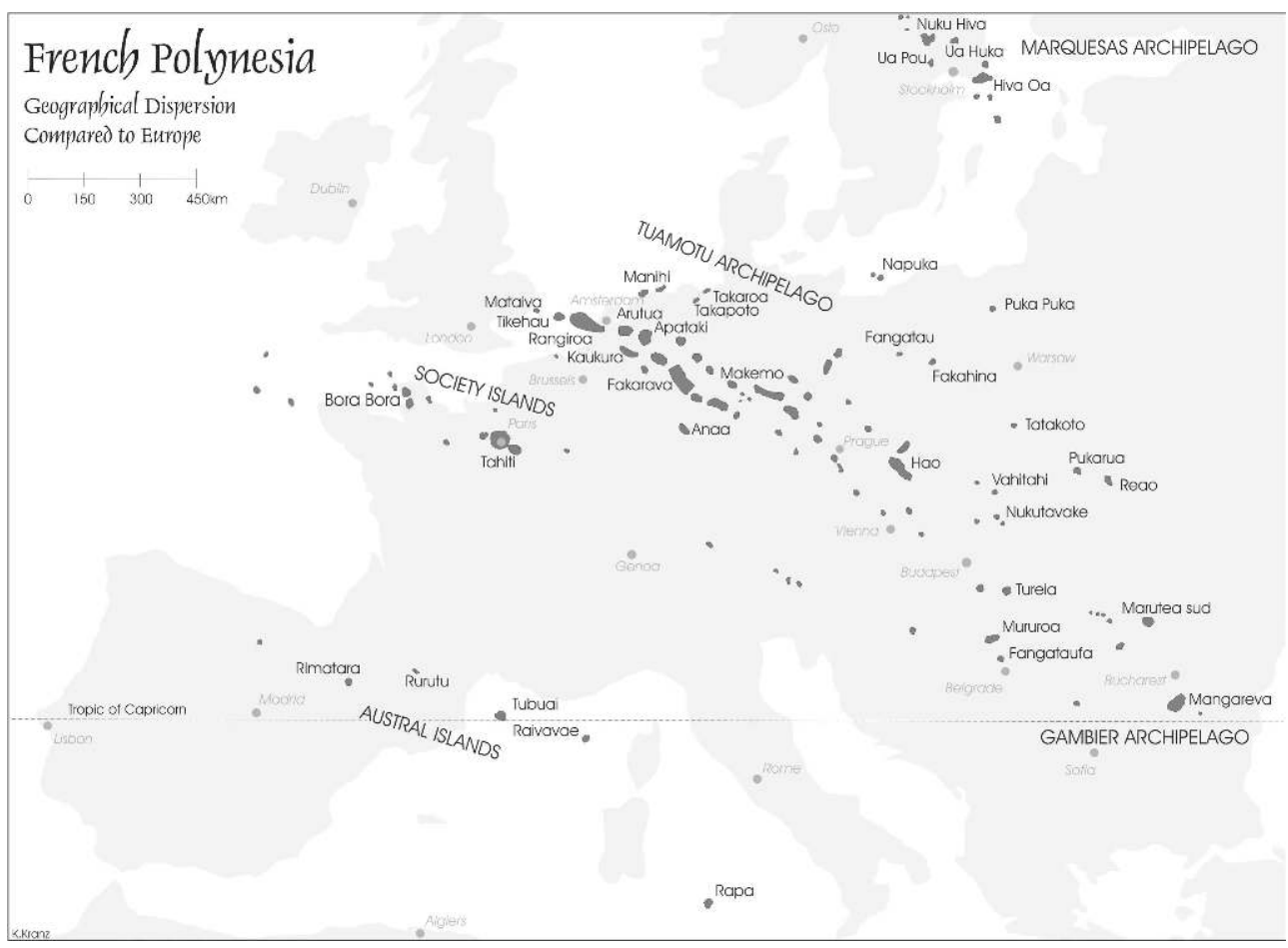

6 Le plan de développement économique repose sur la diversification des activités pour assurer leur durabilité : tourisme et artisanat en sont deux piliers, auxquels il convient d'ajouter les fermes perlières dans les Tuamotu-Gambier, l'agriculture dans les Australes et la pêche hauturière aux Marquises, sans compter les activités traditionnelles de pêche lagunaire et de production de coprah et de vanille. Nous n'examinons ici qu'un des objectifs de ce plan qui est de provoquer le retour dans les archipels de leurs émigrés pour réduire la taille d'un sous-prolétariat, dû, pense le gouvernement, à leur expatriation. Trois mille « Marquisiens » vivent à Tahiti contre 8 700 seulement aux Marquises. Les Tuamotu qui contenaient $11 \%$ de la population du territoire en 1962 n'en comptaient plus que 6,8\% en 1967, pourcentage qui s'est stabilisé depuis.

7 Une des questions qui se pose aujourd'hui est de savoir quelle forme et quelles opportunités viendront du nouveau paysage informatique et comment les communautés de la Polynésie française vont se situer par rapport à cette évolution. Alors que l'Internet est devenu un outil indispensabe pour demeurer compétitif sur le marché national et international, ce qu'ont déjà compris certains entrepreneurs dans les archipels, on peut se demander dans quelle mesure ce n'est pas l'avenir des archipels et de leurs résidents et l'amorce du retour de leurs émigrés. Le gouvernement envisage le recours aux nouvelles technologies comme le moyen qui va donner à cette nation (d'autant que ce n'est pas un état indépendant) le pouvoir de se placer sur la scène globale, tout en facilitant le désenclavement de ses archipels éloignés. 


\section{Terrain d'enquêtes et méthodologie}

8 La Polynésie (ou Te Aho Maohi; la terre des Polynésiens) est une région articulée de l'intérieur par les pratiques quotidiennes de ses résidents (246000 en 2002). Les terres émergées n'occupent que $3521 \mathrm{~km}^{2}$ répartis en 118 îles, dont seulement 76 sont habitées, à l'intérieur d'un territoire océanique de 5,5 millions de $\mathrm{km}^{2}$ (figure 2). Les îles de Te Aho Maohi sont regroupées en cinq archipels dont celui de la Société (îles du vent et îles sous le vent : 215000 habitants) où se trouvent Tahiti et d'autres destinations touristiques bien connues comme Moorea ou Bora Bora. Cet archipel qui représente, dans cette étude, le "centre » de la Polynésie française regroupe $86 \%$ de la population et $83 \%$ des emplois du territoire (Institut Territorial de la Statistique, 1998a). Cette zone a également bénéficié d'équipements complets et d'infrastructures modernes. Et l'on peut s'étonner du fait qu'il reste encore des habitants dans les quatre autres archipels composant la "périphérie»: les Marquises, les Australes et les Gambier qui contiennent surtout des îles hautes, et les Tuamotu composés d'atolls.

Figure 2 : Les archipels de la Polynésie Française

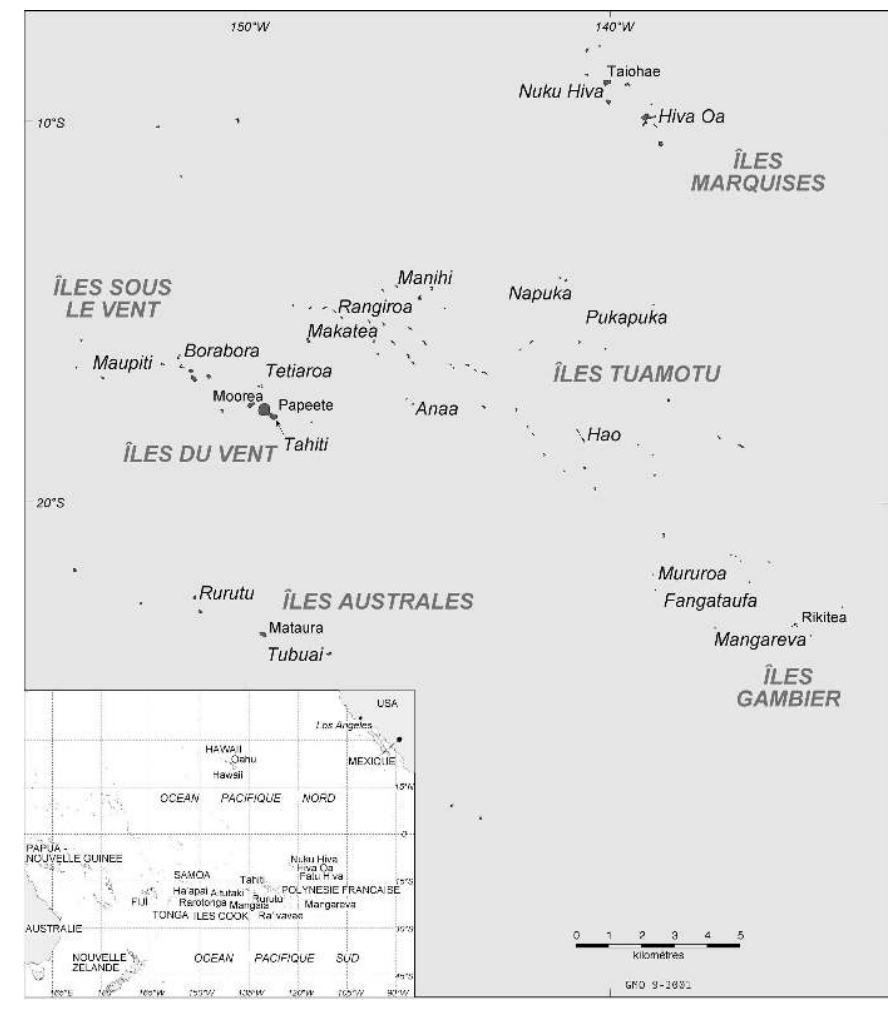

Les six îles principales de l'archipel des Marquises se situent à 1400 kilomètres au nordest de Tahiti. Elles abritent $3,7 \%$ de la population ( 8700 personnes) et 3,3\% des emplois. L'agriculture, dont l'élevage extensif, est l'activité principale. L'artisanat traditionnel, dont la sculpture et le tatouage, mais aussi la confection et la décoration du tapa, est toujours pratiqué. L'archipel volcanique des Australes qui se situe à environ $600 \mathrm{~km}$ au sud sud-est du centre comprend cinq îles d'une superficie totale de $175 \mathrm{~km}^{2}$. $3 \%$ de la population du territoire (6400 personnes) y vivent essentiellement d'agriculture maraîchère et vivrière, favorisée par un climat tempéré. On y trouve le taux le plus élevé de chômage à 15\% (Institut Territorial de la Statistique, 1998b, 1998c). 
10 L'archipel des Gambier à $1700 \mathrm{~km}$ au sud-est du centre abrite $0,4 \%$ de la population du territoire. Il comprend un ensemble d'îles volcaniques et un petit atoll. L'agriculture (surtout vivrière) fut longtemps la seule occupation. Aujourd'hui on y trouve d'importantes fermes perlières. Les atolls de l'archipel des Tuamotu sont disposés en deux lignes parallèles de presque $1200 \mathrm{~km}$ de long entre les latitudes 14 et 23 degrés sud. L'archipel contient 80 îles dont la moitié seulement sont habitées. Elles abritent $6,5 \%$ de la population du territoire ainsi que $6,4 \%$ de ses emplois. Les principales activités économiques sont le tourisme, la culture de la perle noire et la préparation du coprah. Les Tuamotu-Gambier regroupaient 16000 habitants en 2002 (Institut Territorial de la Statistique, 1998d).

11 La méthodologie que nous avons mise en œuvre est qualitative : elle se base sur une lecture critique de textes officiels, de rapports économiques, d'articles sur les migrations et de statistiques ainsi que sur des interviews de résidents de la Polynésie française. Dans cette région, où les habitants sont restreints en nombre et dispersés dans de nombreuses îles difficiles d'accès, les coûts d'obtention de bases d'échantillonnage sont prohibitifs. Les personnes interrogées ont donc été choisies pour être aussi représentatives que possible, bien que l'échantillonnage au hasard ne soit pas garanti. En effet, ces personnes, des deux sexes et de tous âges (le/la plus jeune a seize ans), appartiennent à tous les milieux sociaux, de ministres jusqu'aux plus démunis et proviennent des divers archipels. Elles ont été interpellées dans diverses parties de Tahiti ou dans les archipels éloignés. Les lettres utilisées dans le texte pour les distinguer se rapportent au lieu spécifique des rencontres.

Bien qu'une ou deux personnes seulement soient citées en exemple dans le texte, elles illustrent un point de vue partagé par un plus grand nombre. Environ cent personnes en tout furent interrogées lors de deux séjours en Polynésie en 2001, grâce à une subvention de l'université du Waikato et une aide du gouvernement territorial. La plupart des interviewés sont des migrants en provenance des archipels extérieurs, ou y sont retournés. Nous mettons l'accent sur les représentations ${ }^{1}$ de ces différents résidents de la Polynésie française.

\section{Migrations polynésiennes}

13 L'océan, plus encore que les îles, a toujours été intégré dans la définition du "pays » par les Polynésiens comme le note Teiawa: «nous fondons notre identité dans le Pacifique, aussi large et fluide que soit cet espace» (2001: 343). Cette incorporation permet de concevoir que les cultures océaniennes soient multi-locales (Bedford, 1997), constituant «une corporation de parenté transnationale» (Bertram et Waters, 1985 : 499) qui élargit ainsi les opportunités pour l'amélioration du mode de vie des migrants et/ou de leurs descendants. Les écrits récents en matière de migration ont plus facilement incorporé le nouveau paradigme proposé par Hau'ofa (1994) de percevoir l'océan Pacifique comme une grande route plutôt que comme une barrière. Il est en effet essentiel d'examiner les problèmes de mobilité dans le Pacifique non pas dans les intervalles qui séparent des points de départ et d'arrivée fixes mais comme le résultat d'une façon d'être dans le monde.

Gegeo (2001) souligne que l'identité native peut être "transportée». Il y a, en effet, rarement rupture entre les migrants et leurs lieux d'origine. Les îliens sont des cosmopolites qui habitent des mondes divers et dont l'identité a été construite par 
leurs expériences dans plus d'un environnement géographique, culturel et socioéconomique. Les migrants du Pacifique ont généralement tissé des liens étroits entre les lieux d'origine et ceux d'accueil (Ward, 1997). Les mouvements circulatoires prédominent même si parfois ils doivent s'échelonner sur une ou plusieurs générations. On devrait plutôt parler des migrations des résidents des archipels éloignés comme existant sur une "surface de mobilité " (Bell et Ward, 2000 : 93). Celle-ci inclut les mouvements vers et en provenance aussi bien qu'entre les origines et les destinations. Les formes polynésiennes d'occupation de ce monde océanique se font par des séries d'enchevêtrements culturels, de relations socio-spatiales horizontales qui ont contribué et contribuent encore au tissage de la « communauté Pacifique ».

Les migrations internes actuelles de Polynésie se déroulent dans un contexte mondial de mobilité et plus particulièrement dans un contexte Pacifique. Les Polynésiens appartiennent à une tradition de migration. Le thème du voyage est le ressort permanent et profond de la civilisation polynésienne: "C'est une ancienne tradition insulaire » affirment McCall et Connell (1993). Les Polynésiens, ainsi que l'ont démontré les résultats de fouilles archéologiques, n'ont pas « échoué » sur des îles rencontrées au hasard de dérives bien que certains auteurs aient suggéré que les découvertes se firent au hasard d'expéditions de pêche. Encore fallait-il que leurs connaissances en navigation soient suffisamment solides pour trouver le chemin du retour. Les Polynésiens se déplaçaient avec précision d'îles en îles, pas nécessairement voisines (Kirch, 1986). Rolett (1996) affirme qu'il «existe suffisamment d'évidence ethnobotanique et archéologique pour soutenir l'hypothèse d'une région polynésienne orientale reliée par des voyages de longues distances ».

Les Polynésiens conçoivent leurs domaines comme des relais à travers une mer d'îles dans une région qu'ils ont créée par des pratiques anciennes et modernes de voyages, de visites, d'échanges et de migrations (Hau'ofa, 1994). L'Océanie a une longue tradition de transnationalisme et de création de «méta-sociétés » (McPherson, 1997; Bedford et al., 2001). Les différences étaient juxtaposées et pas contestées, de sorte qu'il ne se créa pas cette association occidentale entre différence et séparation. Les connexions, les routes continuèrent d'être favorisées par rapport aux racines et à la demeure fixe (Clifford, 1997). Le problème pour les Polynésiens ne revenait pas à renier une culture ou à en embrasser une autre, bien qu'au cours des derniers deux cents ans les puissances colonisatrices aient fait des efforts importants dans ces deux sens. Le « culturel » demeure contextuel même si ce contexte est étiré, déplié ou ouvert à des flux de gens, d'idées et d'objets. Les déplacements n'ont pas causé de rupture sociale, culturelle ni donc identitaire. On ne peut dès lors pas accuser ces migrants d'avoir perdu leur indigénéité ou leur capacité de retour.

17 Toutes les sociétés du Pacifique ont évolué avec les migrations. On trouve par exemple 800000 Néozélandais installés dans le monde, alors que la population totale du pays n'est que de 3,6millions, dont 17,5\% sont nés ailleurs (Statistics New Zealand, 1998). Pour beaucoup de jeunes gens, émigrer est devenu un rite de passage (Reichert, 1982). Pour Hau'ofa «[...] le processus contemporain pratiqué par des dizaines de milliers d'îliens efface toutes les frontières économiques et nationales imposées récemment, entrecroisant leurs chemins sur un océan illimité depuis bien avant l'apothéose du Capitaine Cook» (1994: 151). Les migrations ne se sont jamais conjuguées au seul masculin. Les femmes sont des participantes actives dans les décisions des ménages. 
Elles ne sont pas de simples «membres associés », même quand elles choisissent de ne pas émigrer.

On ne peut donc pas parler de contraste entre un passé (pré) colonial marqué par la fixité des populations indigènes et une mobilité post-coloniale, entre des indigènes traditionalistes enracinés et des exilés multiculturels qui seraient cosmopolites et modernes (Chapman, 1995). Les circuits contemporains de mobilité des Polynésiens reproduisent ceux du passé; ils n'ont pas été, contrairement aux suggestions de certains écrivains (Connell, 1990; Zelinsky, 1991), amorcés par les contacts avec le monde occidental ni les dislocations que peuvent engendrer la modernisation ou la globalisation de l'économie locale. Aucun des archipels n'a souffert, entre 1988 et 1996, du seul exode de sa population et aucun n'a vu sa population décroître (figure 3 : les données de 2002 ne seront publiées que courant 2004). Le solde migratoire reste négatif pour les Marquises et les Australes, mais malgré cela les Marquises ont vu leur population s'accroître depuis 1977. Seules les Australes n'enregistrent aucune augmentation depuis 1983. Elles maintiennent cependant leur population avec cependant un solde migratoire négatif de 397 personnes entre 1988 et 1996 (Institut Territorial de la Statistique, 1998b, 1998c et 1998d).

Figure 3 : Mouvements migratoires en Polynésie Française

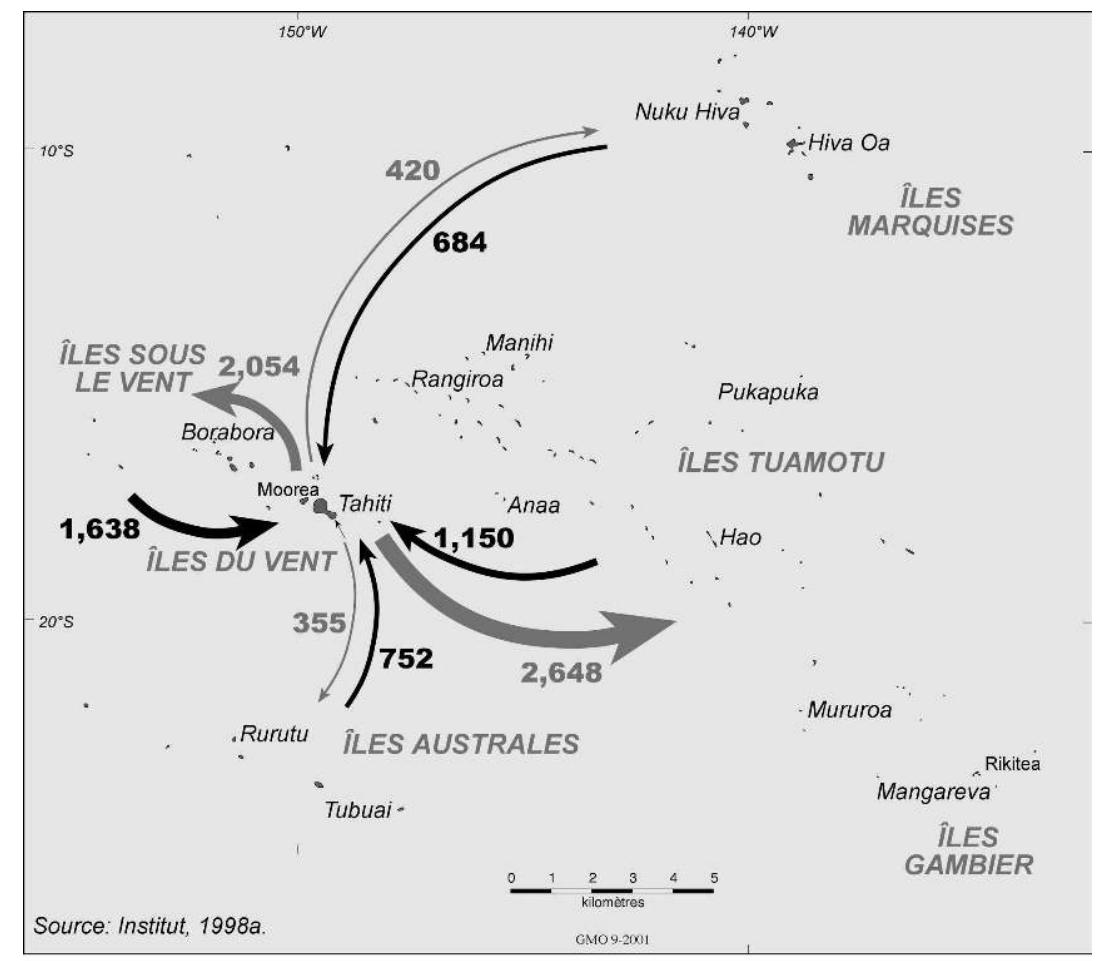

Cependant on ne doit pas passer sous silence le fait que certains mouvements aient été engendrés par des dynamiques politico-économiques, historiques et contemporaines, ainsi que le confirment Domenach et Picouet : «l'emprise politico-institutionnelle des gouvernements métropolitains a été déterminante sur le développement et l'évolution de ces flux » (1992: 33). Beaucoup de leurs habitants ont rejoint leurs "métropoles » à la suite de la deuxième guerre mondiale ou de projets de recrutement de travailleurs en France, au Danemark, aux Pays-Bas et aux États-Unis : 22000 Tahitiens vivent en 
France, et les Tahitiens représentent $2,6 \%$ de la population de Nouvelle-Calédonie (Logan et Cole, 1997). La citoyenneté française garantit l'accès aux lieux et aux marchés du travail à travers l'Union Européenne. De nombreuses familles prévoient ainsi délibérément et stratégiquement où assurer l'éducation (et l'emploi) de certains membres de la famille.

Les pionniers ont établi des réseaux qui entretiennent la pérennité de ces mouvements humains créant un "système de migration du Pacifique " (Massey et al., 1998) qui confirmerait la théorie de causalité cumulative (Massey, 1990). Cette théorie affirme que l'émigration à partir d'une région émettrice tend à se perpétuer car elle en transforme le contexte socio-économique, ce qui encourage plus d'émigration. Le fait semble confirmé dans les archipels éloignés d'où les départs continuent malgré les améliorations des services (enseignement, santé, etc.) et de l'infrastructure (transports et communications).

\section{Autres courants migratoires}

21 Des Européens, des Arorai (habitants de Kiribati), des Chinois et des Japonais vivent à Tahiti et dans ses îles, certains depuis plusieurs générations. Ces flux migratoires pèsent d'un poids considérable à cause de la taille réduite de la population originelle. Ils illustrent eux aussi la fluidité démographique en Polynésie et à travers le Pacifique et confirment la nature transnationale de la culture polynésienne. Les Européens ont abordé la Polynésie dès 1595 , et de façon régulière après 1765 . Ne pouvant les repousser, les Polynésiens décidèrent d'exploiter leur présence qui avait, entre autres, l'avantage de leur offrir l'éclaircissement du teint et la diversification génétique de leurs futurs descendants. La présence coloniale continue fut mal acceptée bien qu'aucun courant migratoire en provenance de France ne s'établît: les seuls colons furent des militaires démobilisés sur place ou des étrangers (quelques Anglo-Saxons et Allemands). Mais l'opposition, tout au cours de l'histoire, est demeurée dispersée et mal structurée.

Le CEP a entraîné, à partir de 1964, la présence de 13000 métropolitains de plus et des rapports tendus entre des Français de passage (les séjours ne duraient que 2 à 6 ans) et les résidents locaux (Toullelan, 1991 ; Doumenge, 1999). Aujourd'hui, les Français et les Chinois totalisent $16 \%$ de la population mais occupent $23 \%$ des emplois! Comme les Tahitiens refusaient de travailler dans les plantations et que leurs nombres décroissaient à cause des ravages des maladies introduites, les colons (métis ou européens) ont cherché ailleurs la main-d'œuvre requise. De 1867 à 1888, 948 Arorai (habitants de Kiribati) quittèrent leur patrie pour travailler d'abord à Atimaono puis sur les plantations des Marquises. Les nombreux migrants chinois ne furent, eux aussi, que temporaires: la plupart de ceux qui travaillèrent dans la plantation de coton d'Atimaono (environ 1100) à partir de 1865 retournèrent en Chine. On déduit de la présence des restes d'un temple qu'un petit nombre de Chinois s'était déjà installé, temporairement, en Polynésie, avant 1850. La majorité des Chinois de Polynésie sont arrivés plus tard, en diverses vagues de migration volontaire, jusqu'en 1949 (Copenrath, 1967).

23 Un autre exemple de dynamique politico-économique et démographique concerne les quelque 1000 personnes qui avaient émigré vers Makatea et y étaient installées dans les années 1950 pour l'extraction de phosphate. Cette exploitation cessa en 1966. Des 
Japonais, puis des Chinois, et enfin des Vietnamiens (environ 300) avaient été recrutés, ainsi que des Polynésiens du territoire français et des îles Cook. En 1996 il n'y restait plus que 84 habitants (Institut Territorial de la Statistique, 1998d). Pendant la deuxième guerre mondiale, Bora Bora fut occupé par des troupes américaines. Aujourd'hui, les fermes perlières des Tuamotu n'arrivent pas à attirer suffisamment de Polynésiens et emploient des greffeurs qui furent d'abord japonais, et maintenant chinois renforçant, même aujourd'hui, l'hétérogénéité et le métissage de la population, tout en continuant cette tradition de flux migratoires.

Le tourisme dépend de la capacité de déplacement d'individus de leur lieu de domicile vers le lieu de distraction. C'est une forme de migration internationale, temporaire certes, mais certainement une force majeure aujourd'hui, qui se continue à l'intérieur de la Polynésie en direction de diverses îles ou archipels plus ou moins éloignés selon les motivations de ces touristes. Elle se double, à l'intérieur de la Polynésie, des mouvements de touristes domestiques. Plusieurs Européen(ne)s, après avoir connu ces îles en tant que visiteurs, s'y sont installé(e)s pour servir les touristes. Certain(e)s ont épousé des personnes originaires de l'île où ils vivent. Comme l'affirme Papastergiadis les « migrations... n'ont pas une origine unique ni une simple fin » $(2000: 2)$.

\section{Motivations des migrants}

La décision d'émigrer (et cela sous-entend celle de retourner) est souvent une réponse active à des stimuli intrinsèques aussi bien qu'extrinsèques. Les évènements migratoires existent dans le flux biographique et dans les échanges de connaissance d'individus et de générations d'individus : « [...] il y a toujours une autre histoire... une certaine volonté, un empressement même à innover dans le mode de vie » (Jorgensen, 1983). Les hommes en âge de travailler ne sont pas non plus les seuls à avoir émigré comme le confirme Simon (1995: 30). La rébellion et le désir de s'échapper sont des motivations fortes pour de nombreuses jeunes émigrées (Kincaid, 1985; Chamberlain, 1997 ; Hughes, 1999). Mathilde (HA 10) a quitté les Marquises car elle ne voulait pas d'un mariage arrangé. Une autre (I2) est partie car elle n'avait pas le droit de fréquenter qui que ce soit en dehors de sa famille. Ces personnes, comme Riorita (cidessous), cherchaient plus de liberté, une forme non économique d'amélioration du mode de vie.

Riorita, une jeune métisse sino-polynésienne avait quitté Tubuai (dans l'archipel des Australes) et trouvé un emploi comme assistante aux travaux ménagers dans une famille française. Au bout de 10 mois d'emploi, elle demanda à pouvoir rentrer à Tubuai pour participer à la préparation du coprah. Autorisation lui fut donnée. L'année suivante, Riorita redemanda un congé. Sa précédente absence ayant duré trois mois, l'autorisation lui fut refusée. Riorita de joie, sauta au cou de sa patronne. La famille de Riorita ne manquait pas de ressources ni de bras: la famille admettait qu'elle est obligée d'obéir aux exigences de sa patronne. Riorita elle, appréciait l'indépendance qui l'avait poussée à émigrer et ne tenait pas à retrouver certaines contraintes familiales même de façon temporaire.

Il serait simpliste d'associer l'émigration à un taux élevé de croissance de la population de la zone émettrice. En Polynésie, cette croissance signifierait une insuffisance de ressources pour subvenir aux besoins de tous les habitants. Les Polynésiens vivaient à des taux d'occupation des îles bien plus élevés avant l'arrivée des Européens puisqu'une 
des conséquences majeures de la colonisation fut le déclin rapide de la population. La population de Mangareva dans les Gambier est passée de 5000 à 500 entre l'époque de sa découverte et le début du vingtième siècle et celle des Marquises a périclité de 43000 en 1800 à 2000 en 1924. La seule île de Nuku Hiva comptait encore 12000 habitants en 1842. Il n'en restait que 635 en 1934 (Rallu, 1990, Institut Territorial de la Statistique, 1998b). Les causes en ont été à la fois les dévastations par maladies, la réduction de la fertilité (elle-même une conséquence d'infection vénérienne importée par les Européens) et les famines provoquées par le manque de pluie. S'y ajoutèrent les déprédations du blackbirding des Péruviens et des guerres intertribales constantes (Maude, 1986). La population des Marquises est aujourd'hui de 8700 personnes et celle des Gambier de 1100. Nuku Hiva compte 2000 habitants (Institut Territorial de la Statistique).

Les motivations des migrants interwievés (originaires de ces archipels éloignés maintenant installés à Tahiti) peuvent être regroupées sous trois rubriques : recherche d'une occupation rémunérée en dehors du secteur primaire, éducation des enfants (audelà de l'école primaire) et violence familiale. Simon souligne que les facteurs familiaux et les études sont devenus des motivations de migration importantes (1995). Les taux de chômage ou de sous-emploi dans les archipels éloignés sont relativement bas. Aux Tuamotu, en 1996, le taux des actifs est de $69,4 \%$ contre une moyenne de $58,1 \%$ en Polynésie. 90\% des hommes entre 25 et 50 ans sont actifs et 63,5\% des femmes (Institut Territorial de la Statistique, 1998a). Cela est dû en partie au mode de vie traditionnel sur des terres encore aux mains des Polynésiens. Quand les enfants atteignent l'âge d'une scolarité extérieure, les parents ont alors besoin de revenus supplémentaires, surtout s'ils accompagnent leurs enfants pour s'assurer de leur succès scolaire. L'émigration des parents est donc alors causée par les besoins éducatifs des enfants, plus que par des motivations personnelles ou simplement économiques. Certains parents, ayant trouvé un travail à Tahiti, ne sont plus retournés dans leur archipel (HA9 et HA11). Ces trois motivations de migration se conjuguent, en fait, de multiples façons.

Beaucoup de jeunes femmes quittent les archipels éloignés pour parfaire leur éducation plus souvent que les garçons. Ces derniers peuvent aider à la culture de la terre, domaine traditionellement réservé aux hommes. A priori, leur accès au statut adulte est un avantage prisé, mais leur faible niveau scolaire retarde leur incorporation dans la société occidentale, limitant leurs options quand ils ont fondé une famille. La réaction des hommes alors, surtout si leur femme est mieux scolarisée et donc plus à même de traiter avec l'administration ou les commerçants, est de boire et d'être violents. Les conséquences de leurs actes peuvent même parfois causer la dislocation de la famille. F4 qui a à peine 16 ans vit depuis cinq ans dans diverses institutions et séparée de ses frères et sœurs depuis le meurtre par son père de sa plus jeune sœur. La violence ne concerne pas uniquement les filles. Les soulèvements de 1987 et 1995 furent causés par des jeunes maltraités, consciemment manipulés par l'opposition politique (Kerdilès, 1998). On a parlé d'inégalités économiques, de chômage, de dédain pour la culture Maohi: ces jeunes manquent de qualifications parce que leurs parents refusèrent de les y préparer (Kerdilès, 1998 : 145).

Les filles bénéficient plus souvent d'une éducation prolongée que les garçons, non seulement parce qu'elles ne peuvent pas aider aux cultures mais sans doute aussi parce que motivées par la violence familiale et sexuelle ambiante: la poursuite de leurs études leur offre une avenue hors de l'enceinte familiale et sociale. Nombre de mes 
interlocutrices fuyaient des modes de vie physiquement inconfortables. Comme l'école (refuge) est dans un autre archipel, les jeunes filles peuvent également plus facilement refuser de retourner trop souvent dans la famille (F6). F2 préférait voyager en Europe et aux États-Unis que de rentrer chez elle aux Marquises pendant les vacances. La violence sexuelle touche aussi les garçons, et les enfants adoptés sont souvent les premières victimes. Les tribunaux de Papeete enregistrent environ une plainte pour viol par semaine, dont les trois quarts concernent des mineurs.

31 Une autre raison citée est la nécessité pour les jeunes en âge de s'installer en ménage de chercher un(e) partenaire ailleurs que sur leur île de naissance, en particulier aux Australes à cause des liens de parenté. Ils doivent même souvent quitter l'archipel pour cela (F3, HA5, HA6). C'est une des raisons mentionnées par beaucoup de femmes qui n'étaient pas parties pour parfaire leur éducation. Le retour alors devient incertain puisque ce ou cette partenaire risque de ne pas vouloir s'installer dans un lieu "isolé " et dont les traditions sont inconnues. Ce fut le cas de cette mère de 11 enfants (HA6) qui tire ses revenus de l'artisanat car « [...] j'aime ce travail-là ». Elle rentrerait bien, car il y a des terres familiales, mais aucun de ses enfants ne veut l'accompagner (ils ont tous un emploi à Tahiti) et elle trouve la vie aux Australes trop dure sans aide.

\section{Circuits migratoires}

On distingue généralement deux types de mouvements migratoires, temporaires ou permanents. La différence est basée sur la conception occidentale linéaire du temps qui n'est pas nécessairement celle des Polynésiens ou autres migrants non européens. Les distances parcourues obéissent rarement à une règle particulière. On pourrait comprendre que toutes les migrations polynésiennes soient nécessairement temporaires puisqu'en enfouissant le placenta et le cordon ombilical du nouveau-né dans la terre ancestrale on établit « un lien indestructible qui obligerait l'enfant devenu adulte à revenir sur la terre familiale, malgré les voyages qu'il [sic!] aurait pu entreprendre » (Panoff, 1970). Nous examinons ici une migration en provenance des archipels éloignés vers le "centre » que les gouvernements présents sur le territoire voudraient transformer en (ou maintenir comme) migration temporaire. Les lois et les contrôles ne peuvent opérer que pour les migrants étrangers internationaux. Seules des incitations bien ciblées encourageront le retour ou le non-départ des résidents des archipels éloignés.

Nombre de ces migrants utilisent, de plus, non point « une résidence habituelle » d'où ils rayonneraient, mais plutôt « un réseau de résidences" (Taylor, 1996). I4 est née à Papeete, de parents Paumutu, temporairement installés sur l'île de Tahiti. Elle vit aux Tuamotu, mais descend à Tahiti dans sa maison (sur un terrain à elle) pour les grandes vacances. Deux de ses enfants habitent avec elle aux Tuamotu, deux sont installés en France et les autres habitent à Tahiti. Tous ses enfants rentrent régulièrement lui rendre visite, mais aucun ne pense revenir s'installer aux Tuamotu (I4). Beaucoup de Polynésiens, comme ces derniers, reviennent dans les îles en vacances, d'autres pour s'y approvisionner en matières premières pour leur artisanat (coquillages aux Tuamutu, pandanus aux Australes). On peut alors les décrire comme des évènements répétitifs de durée variable. D'autres sont occasionnels, comme pour l'enterrement d'une personne qui a marqué la vie du (de la) migrant(e) (I1 pour l'enterrement de sa grand-mère). 


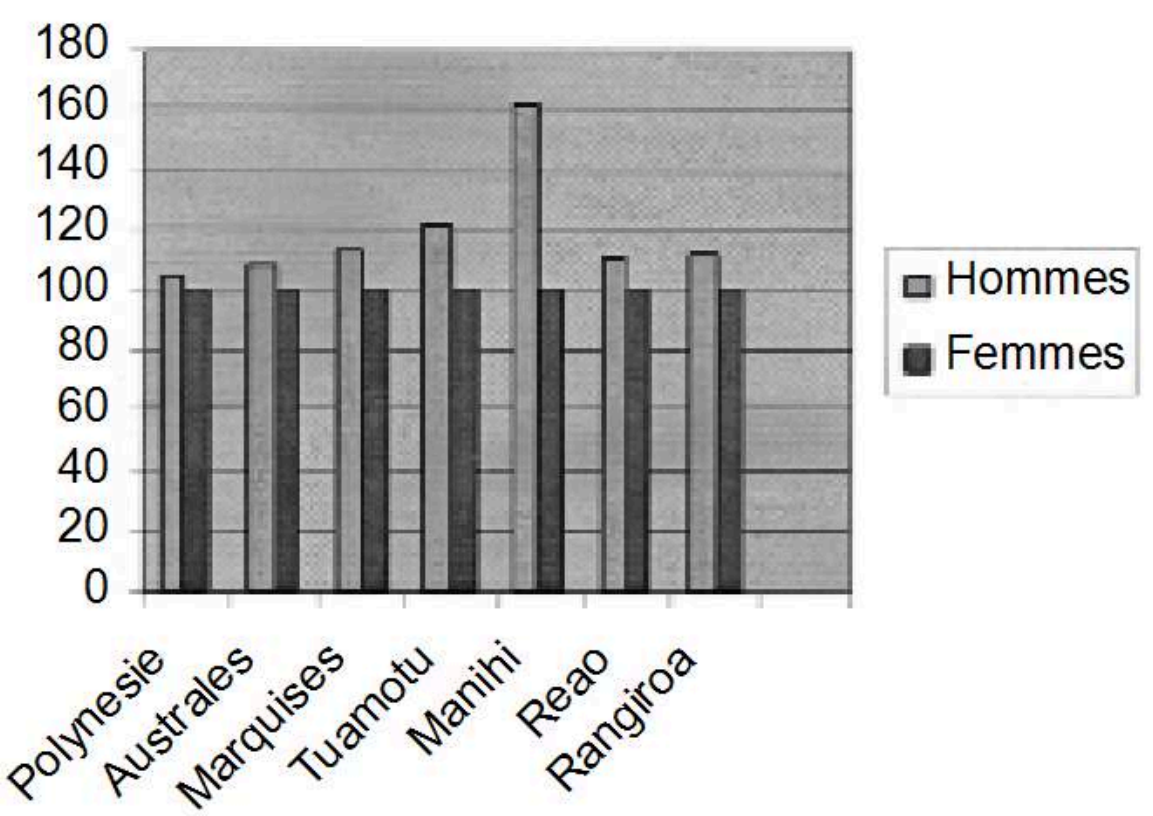

Les mouvements de population internes de Polynésie ne concernent pas uniquement une migration de la périphérie vers le centre ou du rural vers l'urbain. Leur dessin est bien plus complexe. On peut citer l'exemple de Makatea (ci-dessus) ou des atolls de Hikueru et de Takapoto, qui accueillaient tous les ans, dans les années cinquante, cinq à six cents plongeurs en provenance du reste de la Polynésie française et même du Japon. Dans les années soixante, le plastique a remplacé la nacre dans la fabrication des boutons en Europe, mettant fin à cette forme d'interaction économique globale. On note, plus récemment, que les communes des zones urbaines et suburbaines de Tahiti et Moorea ont ressenti une augmentation limitée de leur population (sauf pour Punaauia) alors que celles des zones rurales présentaient une très forte croissance entre 1988 et 1996 (Institut Territorial de la Statistique, 1998a). Les habitants qui ont quitté les archipels extérieurs ne vont donc pas nécessairement vers les quartiers urbains du centre où certains vivaient dans des taudis.

Les Tuamotu et les Gambier ont attiré vers leurs îles un excédent de 1500 migrants sur un flux total de 3800 entre 1988 et 1996 (figure 3). Près de 1000 nouveaux emplois ont été créés grâce en grande partie au développement de la perliculture et du tourisme. On remarque ainsi un accroissement dans les tranches de 20 à 50 ans de 358 personnes (+ 176\%) entre 1988 et 1996 pour les Gambier. Les plus grosses augmentations sont enregistrées pour la classe d'âge des 20 à 30 ans. Ce sont les cohortes qui n'ont pas encore établi de racines ailleurs. Ces personnes considèrent souvent ce déplacement comme temporaire, leur permettant de gagner assez pour s'installer lors de leur retour dans la zone émettrice. Ceci explique que ces 1000 emplois n'aient attiré que 1500 immigrants. Tous ces migrants vers les Tuamotu ne sont pas originaires des îles ni même de l'archipel où sont installés ces emplois. Peu d'entre eux auraient les moyens de créer une ferme perlière ou un service touristique, même s'ils possédaient la terre. Ils sont venus en majorité comme ouvriers. (Institut Territorial de la Statistique, 1998a et d). 


\section{Migrations de retour}

Quel pourcentage demeurera sur place dix ou vingt ans plus tard? Les statistiques ne sont pas encore disponibles pour déterminer le succès de cette implantation de retour, mais elles indiquent que ces îles attirent une majorité d'hommes. Ils sont majoritaires dans toute la Polynésie, mais plus particulièrement dans les Tuamutu Gambier où l'on constate une immigration positive! Le rapport est de 105 hommes pour 100 femmes en Polynésie en général, mais de 109 aux Australes, 113 aux Marquises et 121 aux Tuamotu. La disparité entre les sexes s'accroît, confirmant l'hypothèse d'un mouvement temporaire: sur l'île de Manihi, le rapport de 143 hommes pour 100 femmes en 1988 passe à 161 en 1996 (Institut Territorial de la Statistique, 1998d). Ceci explique en partie la disparité particulièrement marquée entre les deux sexes dans les Tuamotu. Elle n'est que de 111 à Reao où il n'y a aucune culture de perles et de 112 à Rangiroa où l'activité économique est nettement plus diversifiée grâce au tourisme, à la pêche lagonnaire et au coprah.

Parmi tous ces migrants, ne resteront probablement que ceux qui auront voulu ou pu établir une famille sur place et acheter un terrain où l'y installer, et que si des écoles permettent aux enfants de rester. Certains services demeurent problématiques, ce qui peut décourager l'installation permanente. Peter Ringland (2001) confirme que «la plupart des employés restent un an sur le motu (où se trouve sa ferme perlière) puis achètent un terrain pour s'installer ailleurs ». La mère d'un sixième enfant (A3), originaire de Takaroa, et son mari ont répondu voilà huit ans à l'appel d'une ferme perlière de Manihi car le mari, sans formation scolaire, ne trouvait pas assez de travail à Tahiti. Ils ont dû envoyer leur fille aînée sur l'île de Tahiti quand elle a atteint la seconde. Elle a seize ans et n'arrivait pas à se motiver pour ses études. Les parents l'ont rappelée à Manihi où elle travaille également pour la ferme perlière et commence à se rendre compte des avantages d'une scolarité complète sur le marché du travail.

Le retour dans les archipels éloignés, ainsi que l'indique Poirine (1994) et que confirme cette mère de famille (A3) peut servir de soupape contre la formation d'un sousprolétariat dans le "centre » dont le mécontentement pourrait amener de sérieux ennuis politiques, surtout s'il se manifestait de façon violente. Ce raisonnement sousentend que le sous-prolétariat de Tahiti est composé d'émigrés des archipels éloignés, ce qui n'est que partiellement le cas. Ces personnes peuvent maintenant trouver un emploi dans les fermes perlières et le tourisme des Tuamotu-Gambier, même si elles ne sont pas originaires de ces îles, ou elles peuvent retourner sur les terres familiales là ou dans les autres archipels, puisque de nombreux services y ont été installés pour faciliter leur croissance économique. Dans ce cas, il s'agit d'un retour effectivement motivé par des raisons économiques, mais à l'opposer des théories passées.

Le retour semble envisagé, en fait, par deux groupes principaux : ceux qui pensent pouvoir accroître leur retraite en travaillant dans l'artisanat, ou le tourisme, en ouvrant une petite entreprise qui utilise leurs terres, et ceux qui ont suffisamment de revenus et/ou de pouvoir politique pour être inclus dans le cercle qui prend les décisions politiques. Ils ont aussi suffisamment de revenus pour s'échapper quand l'isolement et l'ennui leur pèsent trop. Quelques-uns retournent dans les îles pour suivre un partenaire ou parce qu'ils ont pu trouver une occupation qui est à la hauteur de leurs compétences, mais ces opportunités sont rares. A2, par exemple, rentrait aux Marquises car une place l'attendait mais elle est prête à repartir sur Tahiti si le travail 
ne s'avère pas intéressant. Parmi ceux qui sont retournés, pour hériter d'une affaire ou profiter des nouvelles possibilités pour en monter une, un petit nombre souhaite revenir à Tahiti le plus rapidement possible: souvent cela est dû au fait que seul l'intéressé(e) s'est déplacé(e) vers l'archipel. Le reste de la famille est demeuré à Tahiti. Ces personnes sont encore sur place car elles n'ont trouvé personne de confiance pour diriger leur entreprise.

La plupart des migrants (plus de $80 \%$ des interviewés) n'ont aucune intention de rentrer s'installer dans les archipels dont ils sont originaires. Il leur faudrait reprendre un mode de vie qui ne les attire plus, car beaucoup trop dur et austère, même s'il y a maintenant le téléphone et la télévision. Une des filles du père Paumotu mentionné plus haut confirmait qu'elle «[...] aime bien la clim et le confort de Tahiti ainsi que mon travail» (A6). Schaeffer (2001) parle des «habitudes acquises au fil des années: un corpus de réflexes, de relations, de lieux, de temporalités » qui ancrent les migrants, autant que la famille qui a été fondée, dans le pays d'accueil. Ce corpus a également contribué à la création de l'identité de ces migrants, même si elle a été construite à l'origine dans ces archipels éloignés. Les migrations sont devenues un enjeu majeur de société parce qu'elles remettent en question les modes d'accès à l'espace (et ses ressources) et la participation aux interactions sociales et politiques dans la zone réceptrice.

41 L'impression reste que "toute menace d'une retraite forcée vers un mode de vie autonome de subsistance et une réduction des services entraine immédiatement l'émigration et non l'enthousiasme à la perspective de reprendre le "mode de vie Pacifique" » (Brookfield et Watts, $1988: 36$ ). Piore) indiquait déjà que l'expérience d'un mode de vie différent change les goûts et les motivations. Beaucoup ressentent peu d'appartenance à ces lieux qu'elles (ils) ont parfois dû fuir et où elles (ils) conçoivent difficilement une insertion économique et/ou politique à leur avantage. Même si la décision originale était de partir pour un temps déterminé et un but restreint, le séjour en zone réceptrice peut faire acquérir des coutumes et un mode de vie difficiles à obtenir dans la zone émettrice. Les programmes de construction d'écoles et d'infirmeries ne peuvent que renforcer ce processus cumulatif de migration, puisqu'un niveau plus élevé de scolarisation ne peut qu'accroître les revenus potentiels de l'émigré. Retarder le départ des enfants peut, cependant, contribuer à la sédentarisation de leurs parents.

\section{Conclusion}

Les Polynésiens ont toujours voyagé en suivant, généralement, des trajets circulaires. Le retour dans les archipels éloignés de ceux qui les ont quittés pendant les dernières décennies devrait être, a priori, relativement facile à encourager, ainsi que le démontrent les retours effectués depuis 1977 et plus particulièrement 1988. Qui plus est, l'histoire des migrations en Polynésie française contredit l'affirmation de Massey et al. (1998: 277). L'auteur considère que les migrations ont leur origine dans les transformations sociales, économiques et politiques qui accompagnent la pénétration dans les périphéries de modes d'action capitalistes. Il en est de même pour les dislocations causées par leur colonisation. Ces transformations ont poursuivi ou renforcé une tendance qui existait déjà. La pénétration d'Européens en Polynésie a été suivie par une catastrophe démographique pour les Polynésiens, mais elle n'a pas 
enrayé les mouvements de population (Rallu, 1990). Les migrations effectuées par les Polynésiens sont le résultat de la conjugaison synergétique de plusieurs facteurs que devrait pouvoir expliquer la combinaison de diverses théories.

Une meilleure compréhension des motivations de ces déplacements pourrait faciliter la mise en place de dispositifs pour le retour du plus grand nombre. Cela établirait des réseaux dans ces zones jusqu'alors émettrices, sur le modèle de ceux qui facilitent la réception des émigrés dans les zones de réception internes ou internationales. Rien n'est cependant sûr puisque « les nouveaux efforts par les structures gouvernementales établies pour imposer une discipline et une surveillance des migrants... [ainsi que] les tentatives de contrôler leurs géographies n'ont presque rien accompli » (Connell, 1997 : 215). La communauté émigrée concernée est, cependant, elle-même le produit de combinaisons particulières de circonstances. Ses aspirations et ses actions se sont développées dans son propre cadre de contraintes et d'opportunités. Les migrants interviewés ont généralement démontré que leurs actions cherchaient à parer l'imprévu de façon intentionnelle. Il ne s'agissait pas de mesure palliative temporaire.

Le gouvernement territorial et l'État cherchent à utiliser le développement économique des archipels comme déterminant socio-économique pour faciliter le retour de migrants dans leurs archipels d'origine. Les courants migratoires, vecteurs de changements et moyens d'ajustements entre espaces économiques, devraient se plier au plan du gouvernement. Encore faudrait-il déterminer la réversibilité des flux. L'environnement insulaire polynésien (et tout particulièrement dans les archipels éloignés) relève d'un patrimoine historico-culturel confronté à des contraintes de vulnérabilité et de périphéricité. Les lourds investissements en infrastructure, en communications et même en services de santé et de scolarité ne peuvent véritablement recréer le milieu urbanisé de Tahiti ni des "aires de vie» (Courgeau, 1988) que beaucoup n'ont, dans les circonstances actuelles, aucun désir de quitter. Cette infrastructure de désenclavement représente, cependant, un des éléments-clé de tout effort de développement économique. Elle est également essentielle pour attirer des migrants, y compris d'autres que les expatriés de ces archipels.

\section{BIBLIOGRAPHIE}

BEDFORD R. (1997) International migration, identity and development in Oceania : towards a theoretical synthesis, Paper presented at the Conference on International migration at century's end : trends and issues, Barcelona, Spain : IUSSP (International Union for the Scientific Study of Population).

BEDFORD R., MCPHERSON C. et SPOONLEY P. (2001) « Pacific communities in the Information Age », In V. Naidu, E. Vasta et C. Hawksley, Éds., Current Trends in South Pacific Migration, Wollongong : Migration and Multicultural Studies Program.

BELL M. et WARD G. (2000) « Comparing temporary mobility with permanent migration », Tourism Geographies, 2(1), pp. 87-107. 
BERTRAM I.G. et WATERS R.F. (1986) « The MIRAB economy in South Pacific microstates », Pacific Viewpoint, 26 (3), pp. 497-520.

BERTRAM I.G. et WATERS R.F. (1986) « The MIRAB process : earlier analyses in context » Pacific Viewpoint, 27 (1), pp. 47-59.

BROOKFIELD M. et WATTS G., Eds. (1988) New Directions in the South Pacific : A Message for Australia, Canberra : Academy for the Social Sciences in Australia, Australian National University.

CHAMBERLAIN M. (1997) « Gender and the narratives of migration », History Workshop Journal, 43, pp. 87-108.

CHAPMAN M. (1995) «Island autobiographies of movement : alternative ways of knowing? ». In P. Claval and Singaravelou, Éds., Ethnogéographies, pp. 247-259, Paris : L’Harmattan.

CLIFFORD J. (1997) Routes : Travel and Translation in the late Twentieth Century, Cambridge, MA : Harvard University Press.

CONNELL J. (1990) « Modernity and its discontents : migration and change in the Pacific ». In J. Cohen, Éd., Migration and Development in the South Pacific, 1-28, Canberra : National Center for Development Studies, Australian National University.

CONNELL J. (1990) « A false global-local duality?». In P. J. Rimmer, Éd., Pacific Rim Development. Integration and Globalisation in the Asia-Pacific Economy, Sydney : Allen and Unwin.

COPENRATH G. (1967) Les Chinois de Tahiti, de l'aversion à l'assimilation, 1865-1966, Paris, Société des Océanistes, Musée de l'Homme.

COURGEAU Daniel (1988) Méthodes de mesure de la mobilité spatiale, Paris : Éditions de l'INED.

DOMENACH Hervé et PICOUET M. (1992) La dimension migratoire des Antilles, Paris, Economica.

DOUMENGE J-P. (1999) «L'immigration dans les territoires d'outre-mer : réalités et perceptions ». In J-Y Faberon et Y. Gautier, Éds., Identité, nationalité, citoyenneté outre-mer, 135-152, Paris, la Documentation Française.

ÉTAT-TERRITOIRE (1994) «Contrat de développement État-Territoire 1994-1998 ». Journal Officiel de la Polynésie Française, 143 (1), pp. 2-51.

GEGEO D. (2001) «Cultural rupture and indigeneity : the challenge of (re)visioning "place" in the Pacific », The Contemporary Pacific, 13 (2), pp. 491-509.

HAU'OFA E. (1994) « Our sea of islands », The Contemporary Pacific, 6(1), pp. 147-161.

HUGHES R. (1999) «Between the Devil and a warm blue sea : islands and the migration experience in the fiction of Jamaica Kincaid », In R. King and J. Connell, Éds., Small Worlds, Global Lives : Islands and Migration, London, Pinter.

INSTITUT TERRITORIAL DE LA STATISTIQUE (1998a) Les tableaux de l'économie polynésienne, Papeete : Imprimerie Officielle.

INSTITUT TERRITORIAL DE LA STATISTIQUE (1998b) Îles Marquise : recueil des données essentielles, Papeete : Imprimerie Officielle.

INSTITUT TERRITORIAL DE LA STATISTIQUE (1998c) Îles Australes : recueil des données essentielles, Papeete : Imprimerie Officielle.

INSTITUT TERRITORIAL DE LA STATISTIQUE (1998d) Les Tuamotu Gambier : recueil des données essentielles, Papeete : Imprimerie Officielle.

JOHNSTON R. J. (1988) « There's a place for us ». New Zealand Geographer, 44(1), pp. 8-13. 
JORGENSEN D. (1983) « Introduction : the facts of life, Papua New Guinea style ». Mankind, 14(1), pp. 1-12.

KERDILES M. (1998) Itinéraire polynesien, Papeete : Au Vent Des Iles.

KINCAID J. (1985) Annie John, New York : Plume.

KIRCH P. (1986) « Exchange systems and inter-island contact in the transformation of an island society : the Tikopia case ». In P. V. Kirch, Éd., Island Societies : Archeological Approaches to Evolution and Transformation, 33-41, Cambridge : Cambridge University Press.

LAVENTURE M. (1997) Le tourisme, facteur de développement de l'outre-mer français, Paris : Direction des Journaux Officiels.

LOGAN L. and COLE G. (1997) New Caledonia, Hawthorn, Australia : Lonely Planet.

MASSEY D. (1990) «Social structure, household strategies and the cumulative causation of migration ». Population Index, pp. 3-26.

MASSEY D., ARANGO J., HUGO G., KAOUAOUCI A., PELLEGRINO A. and TAYLO, E. (1998) Worlds in Motion : Understanding International Migration at the End of the Millennium, Oxford : Clarendon Press.

MAUDE H.E. (1986) Slavers in Paradise. The Peruvian Labour Trade in Polynesia 1862-1864, Suva, Fiji : University of the South Pacific.

MCCALL G. and CONNELL J. (1993) « Pacific islander migration : context and prospects ». In G. McCall and J. Connell, Éds., A World Perspective on Pacific Islander Migration : Australia, New Zealand and the USA, Kensington, NSW : University of New South Wales. Center for South Pacific Studies, Pacific Studies Monograph 6.

MacPHERSON C. (1997) «The Polynesian diaspora : new communities and new questions ». In Ken'ichi Sudo and Shuji Yoshida, Éds., Contemporary Migration in Oceania : Diaspora and Network, Osaka : Japan Center for Area Studies.

PALACIOS C. (1987) WWNFIP.

PANOFF M. (1970) La terre et l'organisation sociale en Polynésie, Paris : Payot.

PAPASTERGIADIS N. (2000) The Turbulence of Migration, Cambridge : Polity Press.

PIORE M. (1979) Birds of Passage: Migrant Labor in Industrial Societies, New York : Cambridge University Press.

POIRINE B. (1994) «Tahiti : Is the melting pot melting down? », Pacific Viewpoint, 35(1), pp. 57-82.

PACTE DE PROGRES (1993) Propositions de la délégation polynésienne, Papeete : Éditions Charte du Developpement.

RALLU J.-L. (1990) Les populations océaniennes aux dix-neuvième et au vingtième siècles, Paris : Presses Universitaires de France.

REICHERT J. (1982) « Social stratification in a Mexican sending community : the effect of migration to the United States ", Social Problems, 29, pp. 422-433.

RINGLAND P. (2001) « Mémoires de pionnier », Iles Magazine, 73, pp. 64-65.

ROLETT B. (1996) «Colonisation and cultural change in the Marquesas ». In J. Davidson, G. Irwin, F.Leach, A.Pawley and D.Brown, Éds., Oceanic Culture History. Essays in Honour of Roger Green, Dunedin : New Zealand Journal of Archaeology Special Publication.

SCHAEFFER F. (2001) « Mythe du retour et de réalité de l'entre-deux. La retraite en France ou au Maroc?», Revue Européenne des Migrations Internationales, 17 (1), pp. 165-176. 
SIMON Gildas (1995) Géodynamique des migrations internationales dans le monde, Paris : Presses Universitaires de France.

STATISTICS NEW ZEALAND, TE TARI TARAU, S. N. Z. T. T. (1998). People Born Overseas, Wellington, NZ.

TEIAWA T. (2001) «L(o)osing the edge », The Contemporary Pacific, 13 (2), pp. 343-357.

TOULLELAN P-Y. (1991) Tahiti et ses archipels, Paris : Karthala.

WARD R. G. (1997) « Expanding worlds of Oceania : implications of migration ». In Ken'ichi Sudo and Shuji Yoshida, Éds., Contemporary Migration in Oceania : Diaspora and Network, pp. 179-196, Osaka : The Japan Center for Area Studies.

ZELINSKY W. (1991) « We must not be afraid to ask large questions ». In D. Noin, Éd., Where is Population Geography Going? Paris : International Geographical Union.

\section{NOTES}

1. Les plans des divers gouvernements en matière de migration n'aboutiront que si les individus concernés conçoivent leur avenir en relative homothétie avec celle des dirigeants politiques.

\section{RÉSUMÉS}

Cet article examine les migrations en Polynésie Française en fonction de mesures en faveur du développement économique du territoire prises par le gouvernement de Polynésie et par l'État. Le programme s'est fixé pour objectif de diversifier l'économie des archipels éloignées, qui reste en grande partie basée sur leurs atouts naturels, et à y faire rentrer leurs émigrés. Les migrations que ces mesures espèrent engendrer sont également encouragées par les structures systémiques de production articulées par la nouvelle économie culturelle à l'échelle globale. Les mouvements migratoires relevés aujourd'hui ne diffèrent cependant guère de ceux d'antan. Les Polynésiens se sont toujours déplacés et avaient ainsi déjà établi des réseaux de soutien à la migration à travers le Pacifique. D'après les réponses recueillies lors de récents séjours de recherche en Polynésie l'important effort de construction de nouveaux équipements et d'infrastructure de base dans les archipels ne suffit pas à convaincre les émigrés de rentrer. Cela pourrait, cependant, s'avérer attractif pour des originaires d'autres îles de Polynésie, ne fût-ce que de façon temporaire, comme cela l'est déjà pour certains étrangers.

Economic Planning and Migration in French Polynesia. This article discusses possible ties between migratory patterns in French Polynesia and policies drafted by the French state and the local government concerning the territory's economic development. The goals are to diversify the economic production of the outer archipelagos based on their natural resources and to encourage the return of out-migrants. The structures of production that the new cultural economy has systemically articulated at the global scale should also encourage the return migration that the economic policies seek to establish. Contemporary patterns of mobility differ little from those of times past. Polynesians have always moved and had thus established multiple support networks for migrants across the Pacific. The responses gathered during recent research 
trips to French Polynesia indicate that the large investments in infrastructure by the two governments in the outer archipelagos are not convincing most out-migrants to return. This infrastructure might, however, attract migrants from other parts of French Polynesia even if only temporarily, as is already the case for some foreigners.

Planificación económica y emigración en la Polinesia francesa. Este artículo examina los movimientos migratorios en la Polinesia francesa en función de las medidas que el gobierno de Polinesia y el Estado han tomado en favor del desarrollo económico. El programa se ha fijado como objetivos diversificar la economía de los archipiélagos alejados la cual depende en gran medida de los atractivos naturales, y por otra parte facilitar el retorno de los emigrantes. Las migraciones que estas medidas esperan engendrar derivan igualmente de las estructuras sistémicas de producción articuladas por la nueva economía cultural global. Sin embargo, los movimientos migratorios identificados actualmente no difieren enormemente de sus homólogos del pasado. Los polinesios se han desplazado siempre a lo largo del Pacifico estableciendo redes de apoyo a la emigración. Según las respuestas recogidas recientemente durante varias estancias de investigación en Polinesia, el esfuerzo importante de construcción de nuevos equipamientos e infraestructuras de base en los archipiélagos no constituye, para los emigrantes, una razón suficientemente convincente para volver. Estas infraestructuras podrían, sin embargo, constituir objeto de atracción, incluso de forma temporal, para los habitantes originarios de otras islas de Polinesia. Se trata ya del caso para ciertos extranjeros.

\section{INDEX}

Mots-clés : circulation migratoire, politique économique

Index géographique : Polynésie Française

\section{AUTEUR}

\section{ANNE-MARIE D'HAUTESERRE}

Docteur es Lettres, Tourism Program Coordinator, Department of Geography, University of Waikato, Private Bag 3105, Hamilton, New Zealand. E-mail : adhautes@waikato.ac.nz, Fax : 64-7-8384633 